EPJ Web of Conferences 64, 05006 (2014)

DOI: $10.1051 /$ epjconf/ 20146405006

(C) Owned by the authors, published by EDP Sciences, 2014

\title{
Three-dimensional simulations of MHD disk winds to hundred AU scale from the protostar
}

\author{
Jan Staff ${ }^{1}$, , Nico Koning ${ }^{2}$, Rachid Ouyed ${ }^{2}$, and Ralph Pudritz ${ }^{3,4}$ \\ ${ }^{1}$ Department of Physics and Astronomy, MACQUARIE UNIVERSITY NSW 2109, Australia \\ ${ }^{2}$ Department of Physics and Astronomy, University of Calgary, 2500 University Drive NW, Calgary, Alberta, \\ T2N 1 N4 Canada \\ ${ }^{3}$ Department of Physics and Astronomy, McMaster University, Hamilton ON L8S 4M1, Canada \\ ${ }^{4}$ Origins Institute, ABB 241, McMaster University, Hamilton ON L8S 4M1, Canada
}

\begin{abstract}
We present the results of four, large scale, three-dimensional magnetohydrodynamics simulations of jets launched from a Keplerian accretion disk. The jets are followed from the source out to $90 \mathrm{AU}$, a scale that covers several pixels of HST images of nearby protostellar jets. The four simulations analyzed are for four different initial magnetic field configuration threading the surface of the accretion disk with varying degree of openness of the field lines. Our simulations show that jets are heated along their length by many shocks and we compute the line emission that is produced. We find excellent agreement with the observations and use these diagnostics to discriminate between different magnetic field configurations. A two-component jet emerges in simulations with less open field lines along the disk surface. The two-components are physically and dynamically separated with an inner fast and rotating jet and an outer slow jet. The second component weakens and eventually only one-component jet (i.e. only the inner jet) is obtained for the most open field configurations. In all of our simulations we find that the faster inner component inherits the Keplerian profile and preserves it to large distances from the source. On the other hand, the outer component is associated with velocity gradients mimicking rotation.
\end{abstract}

\section{Introduction}

Astrophysical jets are commonly observed in a range of astrophysical objects consisting of a central object surrounded by an accretion disk (protostars, AGNs, X-ray binaries, etc). Here we focus on protostellar jets. These jets are long, originating from a solar system sized protostar-disk system (AU scale), and extending out to parsec scales. They are highly collimated structures, sometimes even showing increased collimation throughout the jet. The magnetocentrifugal mechanism [1], in which a large scale magnetic field is threading the disk, is a possible explanation for both the launching, acceleration, and collimation of the jet.

Using the Hubble Space Telescope, the most nearby protostellar jets in the Taurus cloud at a distance of $140 \mathrm{pc}$ can be resolved down to $14 \mathrm{AU}$ scale. Our simulation box is $90 \mathrm{AU}$ long, thereby covering several pixels of HST observed jets. This, to our knowledge, is the largest three-dimensional

ae-mail: jan.staff@mq.edu.au

This is an Open Access article distributed under the terms of the Creative Commons Attribution License 2.0, which permits unrestricted use, distribution, and reproduction in any medium, provided the original work is properly cited. 


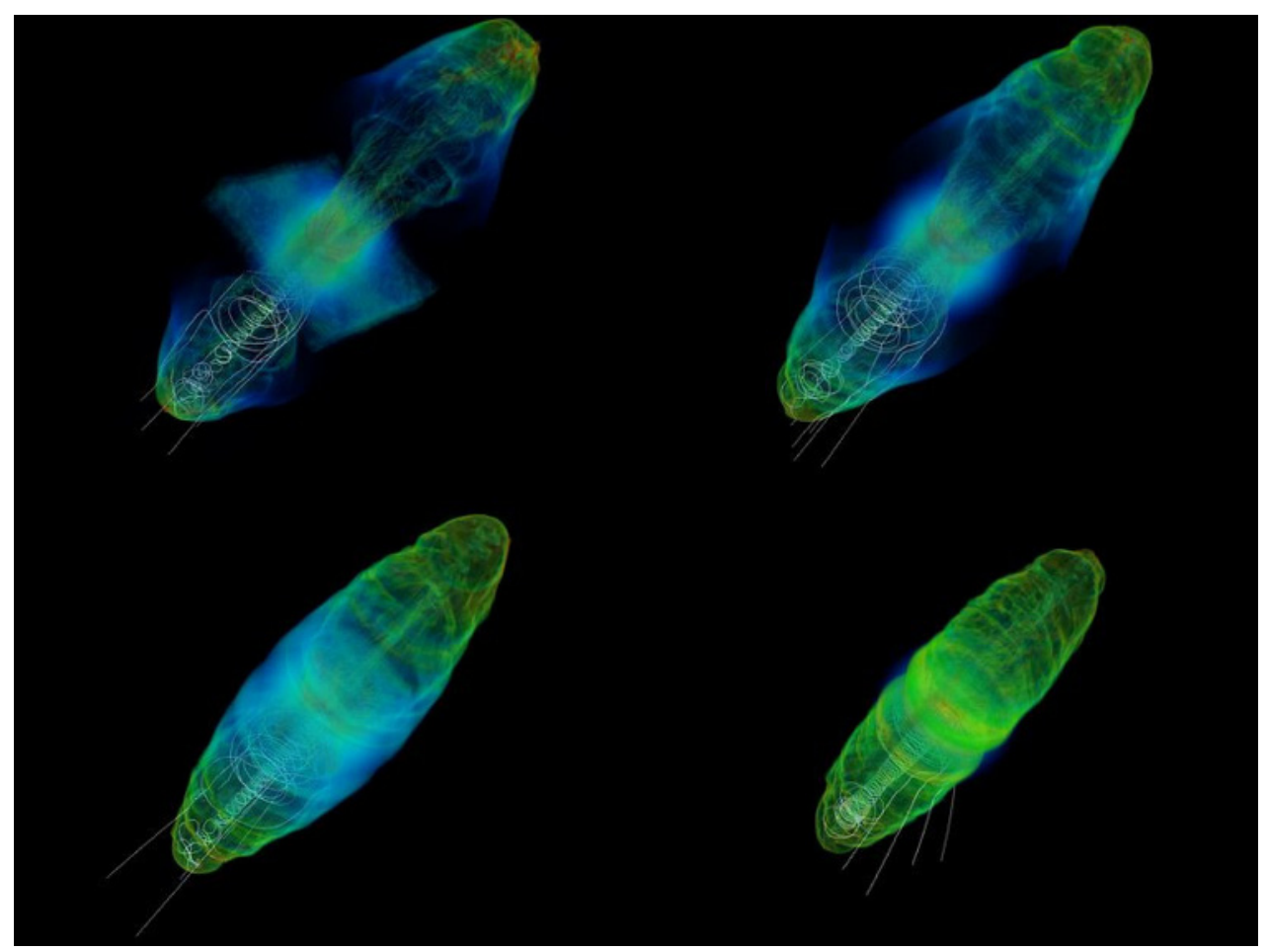

Figure 1. Figure showing the final density structure of the jets resulting from four increasingly open initial magnetic geometries for the disk/halo magnetic field configurations: Upper left panel is the $\mathrm{OP}(\mu=0)$ configuration; Upper right panel is the $\mu=-0.12$ configuration; Bottom left is the $\mathrm{BP}(\mu=-0.25)$ configuration; Bottom right is the $\mathrm{PP}(\mu=-0.5)$ configuration.

protostellar jet simulations performed to date that also resolves the launching region. We have then focused our effort on computing sythetic emission line maps and use these in order to interpret the HST observations of YSO jets. In this work we look for rotation and magnetic structures in simulated jets that lead to features reminiscent of observed jets, features that may be tracked through observations of emission lines.

\section{Methods}

We use the ZeusMP code to run our simulations. The simulation box in all of our simulations extends out to $3000 r_{i}$ along the jet $\left(x_{1}\right)$ direction, and $\pm 900 r_{i}$ in the two transverse directions $\left(x_{2}\right.$ and $\left.x_{3}\right)$; here $r_{i}$ corresponds to $0.03 \mathrm{AU}$. The simulation box is thus $90 \mathrm{AU}$ long and $54 \mathrm{AU}$ wide. The grid size is 1536 zones in the $x_{1}$ direction, and 500 zones in the $x_{2}$ and $x_{3}$ directions. The box size was chosen carefully as to contain the jet within the simulated domain. We stop the simulation once the front of the jet reaches the outer $\left(x_{1}\right)$ edge of the grid.

Details of the numerical setup can be found in [4]. The initial conditions are a point mass at the center of a Keplerian disk with inner radius $r_{i}=0.03 \mathrm{AU}$ and outer radius $r_{\text {out }}=180 r_{i}(6 \mathrm{AU})$, which provide the boundary conditions for the simulations. The outer edge of the disk is much larger than the portion of the disk participating to the outflow; i.e. the size of the launch region. The initial 


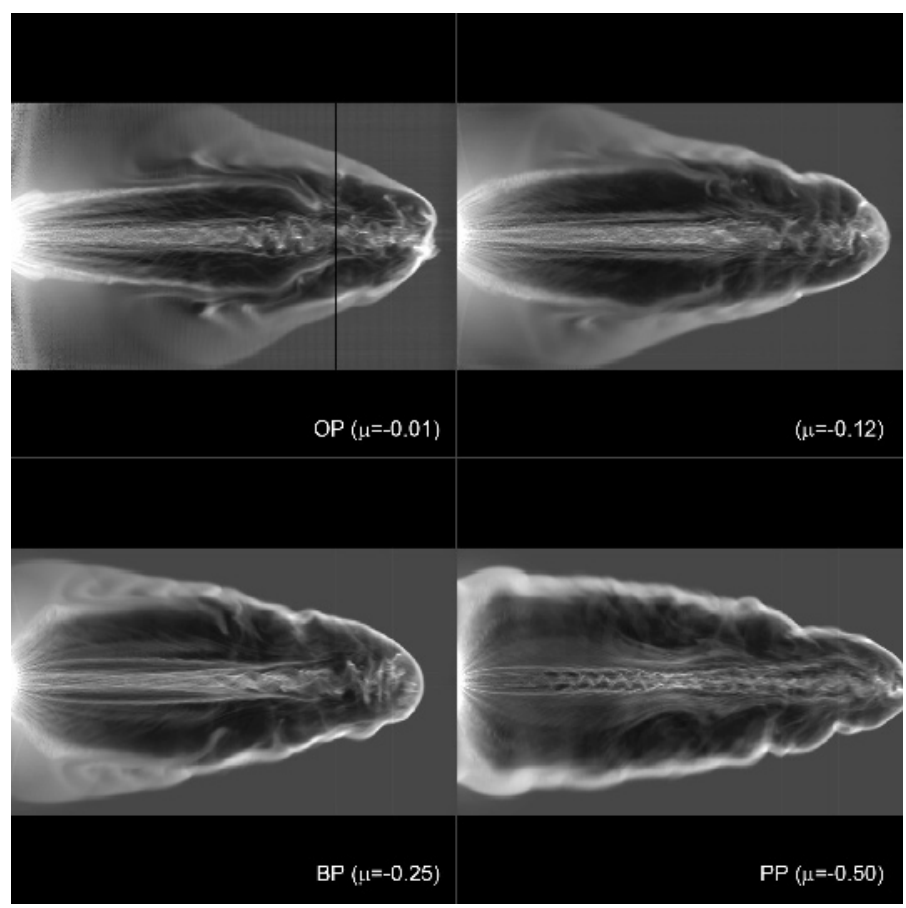

Figure 2. The OP, $\mu=-0.12$, BP, and PP jets seen in MgII $2796 \AA$. The $\mathrm{x}$-axes ranges from 0-90 AU, the y-axis from -45 to $+45 \mathrm{AU}$ for each image.

density profile is that of a hydro-statically stable accretion disk corona, with $\rho \propto r^{-3 / 2}$ for $r<1000 r_{i}$ and constant for $r>1000 r_{i}$ with the two profiles joined smoothly. The disk follows a similar density profile, but it is a factor 100 larger imposed by pressure balance between the disk surface and the overlaying corona.

An initially current-free magnetic field is set up in the corona and extends into the accretion disk [2]. The poloidal magnetic field strength at the surface of the disk falls off as a power laws with disk radius, $B_{p} \propto r^{\mu-1}$. In order to investigate the role of the initial magnetic geometry in launching and collimating disk winds, we set up four different cases; The Ouyed-Pudritz case (with $\mu=-0.01$; hereafter OP), The Blandford-Payne case (with $\mu=-0.25$; hereafter BP), The PelletierPudritz case (with $\mu=-0.5$; hereafter PP), and a fourth case with $\mu=-0.12$ to bridge the gap between the $\mathrm{OP}$ and $\mathrm{BP}$ cases. These range from conditions of most gradually falling and initially somewhat collimated fields to fields which fall off steeply with disk radius and that have much more open magnetic geometries.

To visualize the simulation results, we use the Visit visualization tool (see https://wci.llnl.gov/codes/visit/) which was used to create figure 1 and, the software SHAPE [5] to create the synthetic emission line maps. 


\section{Results}

\subsection{One- and two-component jet structure}

In figure 1, the thin (i.e inner) BP and PP jets are clearly visible, enclosed in a cocoon of material pushed aside by the jet. In the OP and $\mu=-0.12$ simulations, this cocoon is only visible around the front part of the jet, while further back it has been pushed off the side of the grid. In all cases, the jet can be seen to be thightly wrapped in the magnetic field, which collimates the jet.

In Fig. 2 we show the four simulated jets as they would appear in the MgII $2796 \AA$ line. We find that the OP and the $\mu=-0.12$ magnetic field configurations lead to a two-component jet structure. That is, a thin, inner jet surrounded by, and physically separated from, an outer, cylindrical shaped jet. The BP configuration leads to a very weak outer jet (i.e. the second component) and it is seen only close to the source. No second component is seen to develop in PP configurations. Our simulations are suggestive of the BP configuration (the self-similar solutions of disk-winds) separating the onecomponent $(\mu<-0.25)$ from the two-component $(\mu>-0.25)$ regimes. The outer jet appears to recollimate just before terminating. Figure 2 shows that in all cases, the inner jet appears to develop a spiral structure in its outer edge, far from the source (i.e. close to the bow shock). This deformation is more apparent in the PP jet and appears at a location closer to the disk than in the other configurations. The inner jet in the OP and $\mu=-0.12$ simulations appears to open up with a constant opening angle, that we find to be $7.0^{\circ}(\mathrm{OP})$ and $5.6^{\circ}(\mu=-0.12)$. The BP and PP jets do not, instead they appear to re-collimate further out.

Because of the higher Alfvén Mach number ${ }^{1}$ in the BP and PP simulations, the bow shock is bent more sharply backwards in those cases, explaining why the cocoon remains on the grid tightly encapsulating those jets. In the $\mu=-0.12$ simulation, the bow shock is also bent more sharply backwards than in the OP simulation, and the outer jet is therefore terminated earlier upon interacting with the cocoon. Thus, it seems that the shape of the bow shock and the resulting back-flowing current plays a role in the existence and evolution of the second jet component.

\subsection{Jet rotation and jet velocity}

In runs with the less negative $\mu$ (ie. OP and $\mu=-0.12$ ), the resulting jets progress forward faster, with the OP jet being the fastest. The fastest speeds (close to the axis) exceed $400 \mathrm{~km} \mathrm{~s}^{-1}$ while slower speeds are noted closer to the bow shock, as expected. In general, the jets in our simulations have an onion-like velocity structure, with the faster parts closer to the jet axis (see Fig. 3), a feature which seems to have been observed in real jets [3].

In all four configurations, the inner jet inherits the disk's Keplerian profile (see Fig. 4). In that figure, the outer jet in the OP simulation is clearly visible, with a large jump in rotational velocity; this jump in $v_{\mathrm{phi}}$ is another clear indication of the two-component jets. In the $\mathrm{BP}$ case, the rotational velocity does not show such a jump and consists only of one component. We note however that this particular slice is taken in a region far out along the jet direction, not reached by the weak secondcomponent. Nevertheless even taking a slice closer to the disk shows only a weak jump in $v_{\text {phi }}$. The second component (i.e. the outer jet) always rotates slower for more negative $\mu$. In general, while the inner component has a Keplerian profile the outer component does not necessarily and is rather associated with velocity gradients that seem too complex to interpret as a Keplerian profile.

In all cases, the inner jet develops a kin mode (i.e. a spiral-like structure) that becomes prominent at large distances from the source. In the regions of the jets most affected by this instability, the

\footnotetext{
${ }^{1}$ The Alfvén speed is given by: $v_{\mathrm{A}}=\frac{B}{\sqrt{4 \pi \rho}}$, with $\mathrm{B}$ being the magnetic field strength and $\rho$ the gas density, while the Alfvén Mach number is $M_{\mathrm{A}}=v / v_{\mathrm{A}}$, with $v$ being the gas velocity.
} 

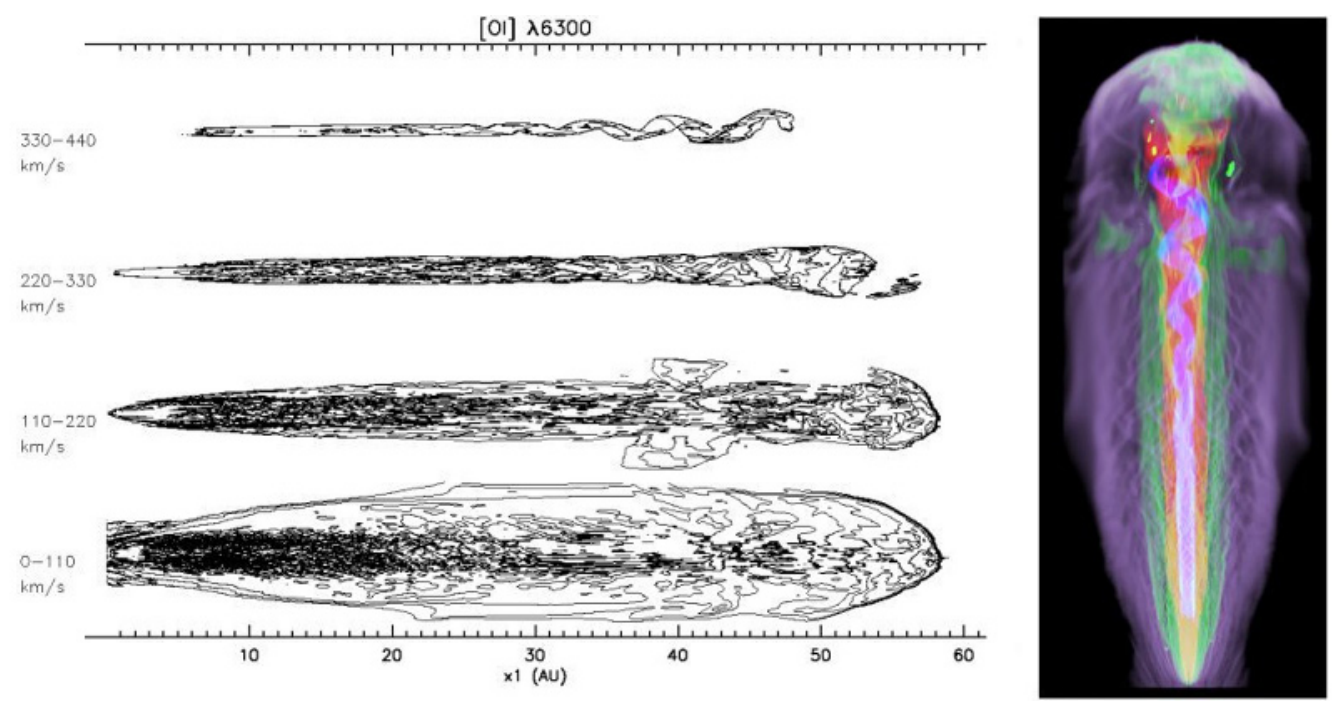

Figure 3. Velocity channel diagram for the OP jet. The four channels are $330-440 \mathrm{~km} \mathrm{~s}^{-1}$ (top), 220-330 $\mathrm{kms}^{-1}$, then $110-220 \mathrm{kms}^{-1}$, and $0-110 \mathrm{kms}^{-1}$ at the bottom. To the right is the same visualized in colors. The fast velocities are found close to the jet axis, while the slower velocities are found farther from the axis. (Figure reproduced from [4]).

Keplerian profile is washed out. However, the Keplerian signature is preserved in most of the length of the jet. The two-component jet in the OP and $\mu=-0.12$ cases do develop such an instability and remain cylindrical to large scales.

In figure 5 we show PV diagrams for all four jets, as seen in the MgII $2796 \AA$ line. The slices are taken at 15, 45, and $75 \mathrm{AU}$ from the disk, respectively. For all jets, except PP, we see at $15 \mathrm{AU}$ and $45 \mathrm{AU}$ signatures of the Keplerian rotation profile in the center. The PP jet also has the highest velocities close to the axis, but as discussed above the spiral structure washes out any signature of the Keplerian rotation profile. Further from the axis, we find an increased velocity for OP and $\mu=-0.12$ at 15 and $45 \mathrm{AU}$, and in $\mathrm{BP}$ at $15 \mathrm{AU}$, associated with the outer jet. At $75 \mathrm{AU}$ the expanding cocoon is dominant in all of the simulations. The cocoon is also visible at $45 \mathrm{AU}$ for all the simulations, and also at $15 \mathrm{AU}$ for BP and PP.

\section{Summary}

Our results show that simulated large-scale MHD disk winds lead to jets with features similar to those observed in real jets. We find that a two-component jet arises in configurations with less open field lines at the disk surface with the inner faster jet inheriting the disk's Keplerian rotation profile. The development of the kink mode and the spiral structure acquired by the inner jets at large distance from the source indicate that signatures of the underlying Keplerian profile may be washed out. However, clean Keplerian profile in the inner jet should be visible closer to the source. The more negative the $\mu$ (i.e. the steeper the poloidal field lines along the disk's surface), the weaker the outer-component with the BP (i.e. the self-similar) configuration seemingly separating the two regimes. For $\mu<-0.25$ only a one-component jet arises in our simulations. We plan to run more simulations with different $\mu$ values in order to to confirm our findings and to understand better these two regimes. 

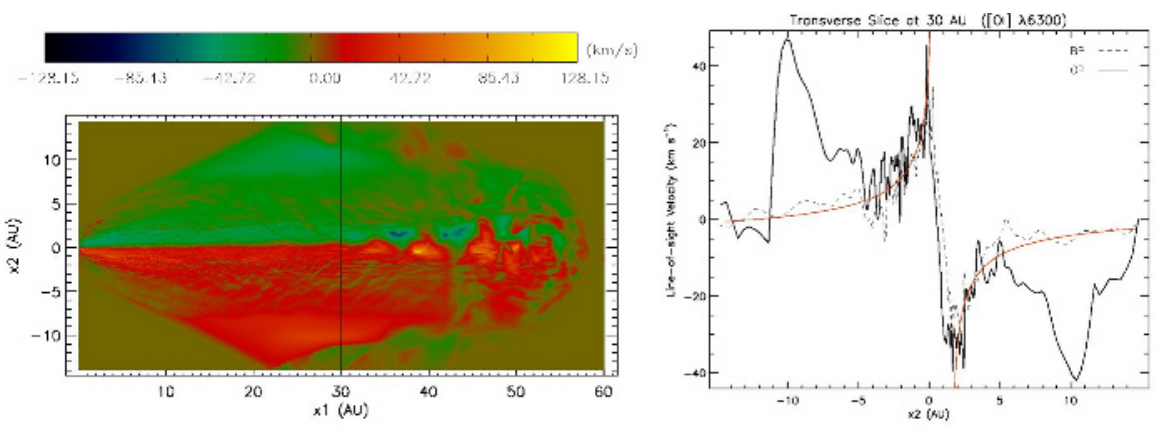

Figure 4. The left panel shows the toroidal velocity inferred from the [OI] line in the OP jet. The right panel shows the toroidal velocity in a cut through the jet at a distance of $30 \mathrm{AU}$ from the disk for the OP jet (solid line) and BP jet (dashed line). The OP jet is seen to strongly deviate from a Keplerian rotation profile (red curve) around $\pm 10 \mathrm{AU}$ from the axis. This is perhaps the best illustration of the 2 jet components. (Figure reproduced from [4]).

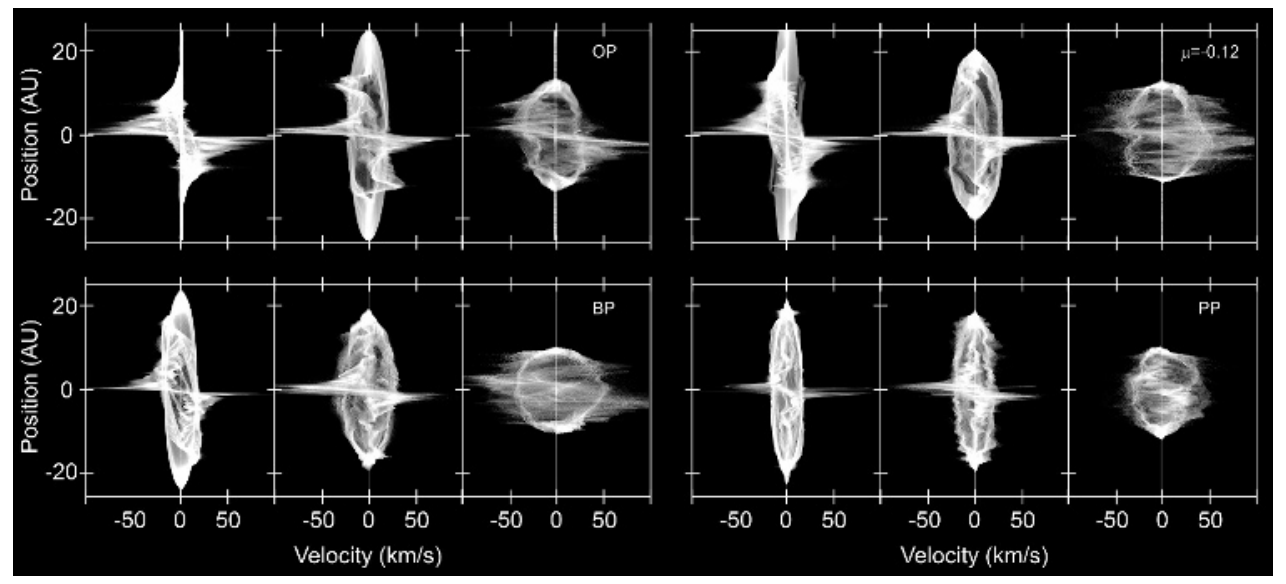

Figure 5. PV diagrams for the OP, $\mu=-0.12$, BP, and PP jets seen in MgII $2796 \AA$ A. For each simulation, the left, middle, and right panel represents the slit locations that are perpendicular to the jet at 15,45 , and $75 \mathrm{AU}$ from the disk.

\section{References}

[1] Blandford, R. D., Payne, D. R. 1982, MNRAS 199, 883.

[2] Jørgensen, M, Ouyed, R., \& Christensen, M, 2001, A\&A 379, 1170

[3] Ray, T., Dougados, C., Bacciotti, F., Eislöffel, J., \& Chrysostomou, A. 2007, Protostars and Planets V, B. Reipurth, D. Jewitt, and K. Keil (eds.), (Tucson: Univ. Arizona Press), 231.

[4] Staff, J. E., Niebergal, B. P., Ouyed, R., Pudritz, R. E., \& Cai, K. 2010, ApJ, 722, 1325

[5] Steffen, W., Koning, N., Wenger, S., Morisset, C., Magnor, M., 2011, IEEE Transactions on Visualization and Computer Graphics, vol. 17, no. 4, pp. 454-465 